Pulmonary, gastrointestinal and urogenital pharmacology

\title{
Pharmacological characterization of a highly selective Rho kinase (ROCK) inhibitor and its therapeutic effects in experimental pulmonary hypertension
}

\author{
Silvia Cantoni ${ }^{a}$, Stefano Cavalli ${ }^{a}$, Fiorella Pastore ${ }^{a}$, Alessandro Accetta ${ }^{a}$, Daniele Pala ${ }^{a}$, \\ Fabio Vaccaro ${ }^{\mathrm{a}}$, Nicola Cesari ${ }^{\mathrm{a}}$, Francesco De Logu ${ }^{\mathrm{b}}$, Romina Nassini ${ }^{\mathrm{b}}$, Gino Villetti ${ }^{\mathrm{a}}$, \\ Fabrizio Facchinetti ${ }^{\text {a,* }}$ \\ ${ }^{\text {a }}$ Corporate Pre-clinical R\&D, Chiesi Farmaceutici S.p.A., Parma, Italy \\ ${ }^{\mathrm{b}}$ Department of Health Sciences, Section of Clinical Pharmacology and Oncology, University of Florence, Florence, Italy
}

A R T I C L E I N F O

\section{Keywords:}

Pulmonary hypertension

Monocrotaline

Rho kinase

Macitentan

Right ventricle systolic pressure

Vascular remodeling

\begin{abstract}
A B S T R A C T
Studies on the role of Rho-associated protein kinase (ROCK) in experimental pulmonary artery hypertension (PAH) relies mainly on the use of pharmacological inhibitors. However, interpreting these data is hampered by the lack of specificity of commonly utilized inhibitors. To fill this gap, we have selected and characterized a novel ROCK inhibitor, Compound 3, previously described in a patent. Inhibitory potency of Compound 3 against enzymatic activity of ROCK- 1 and 2 ( $\mathrm{IC}_{50}=10 \pm 3.1$ and $7.8 \pm 0.5 \mathrm{nM}$, respectively) was accompanied by a strong vasodilating effect in phenylephrine pre-contracted isolated rat pulmonary artery rings $\left(\mathrm{IC}_{50}=\right.$ $51.7 \pm 9.1 \mathrm{nM})$ as well as in aortic rings $\left(\mathrm{IC}_{50}=45.5 \pm 1.1 \mathrm{nM}\right)$. Compound 3 showed a remarkable selectivity towards ROCK 1 and 2 when tested against a large panel $(>400)$ of human kinases. A partial explanation for its selectivity is provided from docking simulations within ROCK-1. Pharmacokinetic studies showed that Compound 3 is suitable for a twice daily administration without significant accumulation upon repeated dosing. In rats with monocrotaline (MCT)-induced pulmonary hypertension, therapy with Compound 3 , ( 1 and $3 \mathrm{mg} / \mathrm{kg}$, s.c., b.i.d.), started 14 days after induction of the disease, attenuated right ventricle systolic pressure (RVSP) increase. Morphometric histological analysis showed that Compound 3, at both doses, counteracted MCT-induced medial thickening of lung distal arterioles with an effect comparable to macitentan (10 mg/kg, p.o., q.d.). Compound 3 is a potent and highly selective ROCK inhibitor that ameliorates hemodynamic parameters and counteracts pulmonary vascular remodeling in experimental PAH.
\end{abstract}

\section{Introduction}

Pulmonary arterial hypertension (PAH) is a rare life-threatening disease characterized by a persistent increase in pulmonary artery pressure $>25 \mathrm{mmHg}$, which is accompanied by augmented vascular resistance and remodeling of the vascular walls of both larger- and smaller-caliber pulmonary artery branches (Schermuly et al., 2011). $\mathrm{PAH}$ treatments relay on the use of prostanoids, endothelin receptor antagonists (ERAs), phosphodiesterase-5 (PDE5) inhibitors and soluble guanylate cyclase stimulators (Galiè et al., 2015). Nevertheless, PAH continues to be a serious clinical problem with high morbidity and mortality.

Rho-associated coiled-coil forming protein kinases (ROCK), which are important down-stream effectors of small GTP-binding protein RhoA, play key roles in pulmonary arterial smooth muscle cells (PASMC) contraction and proliferation (Riento et al., 2003; Feng et al.,
2016). Two isoforms, ROCK-1 and ROCK-2, have been described and found expressed in many human and rodent tissues including the heart, lung and vascular tissue (Oka et al., 2008). ROCK is at the intersection of signal transduction initiated by several agents regulating vascular smooth muscle cell contraction and proliferation, including serotonin, angiotensin II, endothelin I and platelet derived growth factor (Hartmann et al., 2015; Shi and Wei, 2013). Fasudil, one of the first discovered ROCK inhibitor, is characterized by a micromolar affinity for ROCK as well as for other kinases, including PKA, PKG and CaMKII (LoGrasso and Feng, 2009; Tamura et al., 2005). Fasudil has been approved for treatment of cerebral vasospasm after subarachnoid hemorrhage (Satoh et al., 2014) and short-term efficacy of fasudil in the treatment of PAH has been demonstrated in small clinical trials (Raja, 2012). Y-27632 was originally disclosed as a calcium blocker (Muro et al., 1990) and reported to be active as relaxant of vascular and bronchial smooth muscles (Uehata, 1997). Although largely utilized as

\footnotetext{
* Correspondence to: Pharmacology and Toxicology Department, Corporate Pre-Clinical R\&D, Chiesi Farmaceutici S.p.A., Largo Belloli 11/A, 43122 Parma, Italy.

E-mail address: F.Facchinetti@chiesi.com (F. Facchinetti).
} 
<smiles>[X]c1nccc2c(S(=O)(=O)N3CCCNCC3)cccc12</smiles>

1

(Fasudil $\mathrm{X}: \mathrm{H}$ )

1a

(Hydroxyfasudil $\mathrm{X}=\mathrm{OH}$ )<smiles>CC(N)[C@H]1CC[C@H](C(=O)Nc2ccncc2)CC1</smiles>

2

(Y-27632)<smiles>COc1nc(N[C@H]2CC[C@H](N)CC2)c(F)cc1C(N)=O</smiles>

3

(Example 3, WO 2010/032875)

$3^{*} \mathrm{HCl}$

(Example 2, WO 2010/032875)

Fig. 1. Chemical structures of ROCK inhibitors fasudil (1), Y-27632 (2) and Compound 3 (3) as described in the cited patent (WO 2010/032875).

pharmacological tool, Y-27632 shows moderate potency on ROCK and poor selectivity (Ishizaki et al., 2000) and inhibits voltage-dependent potassium channels in arterial smooth muscle cells (Li et al., 2016). Despite their large use, both fasudil and Y-27632 are limited by poor selectivity and low on-target potency resulting in non-specific pharmacological and toxic effects (Li et al., 2016; Asano et al., 1998). Few other ROCK inhibitors have been tested in PAH models. SB-772077-B decreased pulmonary and systemic arterial pressures in rats with MCTinduced pulmonary hypertension, but no data were reported on the effects on vascular remodeling (Dhaliwal et al., 2009). Moreover, relatively poor selectivity was reported for SB-772077-B (Doe et al., 2007). Azaindole-1 (Schirok et al., 2008), an oral ROCK inhibitor, proved to be effective in PAH induced by MCT or chronic hypoxia (Dahal et al., 2010). One limitation of this study is that azaindole-1 was not compared to any of the drugs currently approved for treating PAH patients.

In a series of ROCK inhibitors previously disclosed in a patent application published in 2010, we spotted example 3 (Trans-6-((4-aminocyclohexyl)amino)-5-fluoro-2-methoxynicotinamide), from now reported as Compound 3 (Fig. 1) (Terasawa et al., 2010). Our interest in Compound 3 was elicited by the pharmacological profile reported and by its phys-chem properties similarity to fasudil and Y-27632 that could translate in a similar solubility and bioavailability (Terasawa et al., 2010).

In this paper we report the extensive pharmacological characterization of Compound 3, including potency on target and selectivity, vasorelaxant potency, pharmacokinetic profile and its hemodynamic and anti-remodeling effects in a rat model of MCT-induced PAH. The effect of Compound 3 was compared with macitentan, a dual endothelin-receptor antagonist (ERA) approved for the treatment of PAH (Galiè et al., 2015).

\section{Materials and methods}

\subsection{Synthesis of Compound 3}

Preparation of Compound 3 (Fig. 1) was carried out by adapting the general synthesis procedure reported previously (Terasawa et al., 2010).

\subsection{Docking simulations}

Docking simulations were performed starting from the crystal structure of human ROCK-1 in complex with hydroxyfasudil (PDB 2ETK) (Jacobs et al., 2006). The preparation of the X-ray structure was conducted with the Protein Preparation Wizard tool, applying default parameters. Glide docking grids were centered on the co-crystallized hydroxyfasudil molecule, setting the enclosing and bounding box sizes to $40 \AA$ and $15 \AA$ on each side, respectively. Twenty docking solutions were collected and ranked according to their Emodel score. Only the best-ranked pose of Compound 3 was retained and analyzed.

\subsection{Biochemical enzymatic assays}

Glutathione S-transferase (GST)-tagged 1-535 human ROCK1 and GST-tagged 1-552 human ROCK2 (Fisher Scientific UK Ltd, Loughborough, Leicestershire, UK) were diluted into assay buffer containing $40 \mathrm{mM}$ Tris pH7.5, $20 \mathrm{mM} \mathrm{MgCl}_{2} 0.1 \mathrm{mg} / \mathrm{ml} \mathrm{BSA}, 50 \mu \mathrm{M}$ DTT and $2.5 \mu \mathrm{M}$ peptide substrate (myelin basic protein). Test compounds were dissolved in dimethyl sulphoxide (DMSO) to a final concentration of $1 \%$. All reactions/incubations were performed at $25^{\circ} \mathrm{C}$. Test compounds and either ROCK1 or 2 were mixed and incubated for $30 \mathrm{~min}$. Reactions were initiated by addition of ATP $(10 \mu \mathrm{M})$. After a $1 \mathrm{~h}$ incubation $10 \mu \mathrm{l}$ of ADP-Glo Reagent (Promega UK Ltd, Southampton, UK) was added and after a further $45 \mathrm{~min}$ incubation $20 \mu \mathrm{l}$ of Kinase Detection Buffer added and the mixture incubated for a further $30 \mathrm{~min}$. The luminescent signal was measured on a luminometer. Compounds were tested in dose-response format. To determine the $\mathrm{IC}_{50}$ data were fit to a plot of \% inhibition vs Log10 compound concentration with a sigmoidal fit using ActivityBase software (v 8.05, ID Business Solutions Limited, Guildford, UK).

\subsection{Rat isolated pulmonary artery and aorta assays}

All the experiments were performed according to Gonzales et al. (2000), with modifications. Briefly, Wistar rats were anesthetized with pentobarbital sodium and exsanguinated. Thoracic aortic segments and pulmonary artery were isolated, cleaned of adventitia and aorta was cut into 4 rings (2-3 mm each). Each preparation was placed in 20-ml organ bath filled with oxygenated $\mathrm{CO}_{2} 95 \%$ and $\left.\mathrm{CO}_{2} 5 \%\right)$ normal KrebsHenseleit solution and maintained at $37^{\circ} \mathrm{C}$. Aortic preparations were connected to isometric force transducers under a resting tone of $2 \mathrm{~g}$, pulmonary artery ring under a resting tone of $1 \mathrm{~g}$. After an equilibration period of $60 \mathrm{~min}$, each preparation was contracted with a maximal concentration of phenylephrine (PHE) $(1 \mu \mathrm{M})$. After a thorough washout, vessels were contracted with PHE $(0.1 \mu \mathrm{M})$ and, at the steady tone, a concentration-response curve (CRC) of test substances or vehicle was constructed. Results were expressed as percent of maximal relaxation obtained at the end of CRC by washing the residual PHE-induced contraction.

Some experiments were performed on endothelium-deprived aortic rings under the same experimental conditions. To verify if the 
endothelium was completely removed, carbachol $(10 \mu \mathrm{M})$ was added to the PHE $(0.1 \mu \mathrm{M})$-contracted aortic rings. The lack of any relaxing effect proved the absence of endothelium (Lockette et al., 1986). Subsequently, the preparations were washed out and re-contracted with PHE $(0.1 \mu \mathrm{M})$ and, at the steady-state, CRC of test substances or vehicle were constructed.

\subsection{Animal model of monocrotaline-induced pulmonary hypertension}

All the experimental procedures and conditions involving experimental animals were reviewed and approved by the local ethics committees and authorized by the Italian Ministry of Health (authorization number 33/2016-PRPAH) and were performed in full compliance with the international European ethics standards in conformity to directive 2010/63/EU, the Italian legislative decree 116/1992, and the Animals (Scientific Procedures) Act of 1986. PAH was induced in male Wistar rats (Charles River) with a single subcutaneous injection of MCT $60 \mathrm{mg}$ / $\mathrm{kg}$. The alkaloid MCT (Sigma) was freshly dissolved in $1 \mathrm{~N} \mathrm{HCl}$, and the $\mathrm{pH}$ was adjusted to 7.4 with $1 \mathrm{~N} \mathrm{NaOH}$. Control rats received an equal volume $(1 \mathrm{ml} / \mathrm{kg})$ of isotonic saline. The animals were weighed three times weekly and the weight gain was calculated by subtracting the basal weight to weight at the end of the experiment.

At day 14 post MCT injection rats were randomized into five groups $(\mathrm{n}=10)$ to receive, for two weeks, either saline vehicle $(1 \mathrm{ml} / \mathrm{kg}$, b.i.d., s.c.), 1 or $3 \mathrm{mg} / \mathrm{kg}$ of Compound 3 (b.i.d., s.c.), oral vehicle $(0.5 \%$ carboxymethyl cellulose in water, $10 \mathrm{ml} / \mathrm{kg}$ ) and macitentan $(10 \mathrm{mg}$ / $\mathrm{kg}$, p.o.) once daily.

After 28 days from the MCT induction, rats were subjected to hemodynamic measurements and, subsequently, killed.

\subsection{Measurement of right ventricle pressure and systemic pressure}

On day 28 , test compounds or vehicle were administrated to animals two hours before hemodynamic measurements performed by intra-cardiac catheterization in a blinded manner as described previously (Budas et al., 2018). Systolic arterial pressure (SAP) recording was performed under isoflurane $/ \mathrm{O}_{2}$ anaesthesia with a saline filled polyethylene catheter (PE50) inserted into carotid artery which was connected to a pressure transducer (BLPR, World Precision Instruments, UK). Thoracotomy was carefully performed to expose the heart and the right ventricle systolic pressure (RVSP) was recorded with a Millar catheter. Both Millar catheter and pressure transducer were connected to an amplifier (TBM4M, World Precision Instruments, UK) and an acquisition unit (Powerlab 16S, World Precision Instruments). After stabilization of signal, SAP and RVSP were recorded for at least $15 \mathrm{~min}$. Data were analyzed using a dedicated software, LABCHART (ADInstruments, Australia).

After hemodynamic recordings, animals were euthanized with $5 \%$ isoflurane and by exsanguination. Lungs and hearts were collected for further analyses. The right ventricle free wall (RV) was separated from the left ventricle and septum $(\mathrm{LV}+\mathrm{S})$ to determine the weights and the $\mathrm{RV}$ to $\mathrm{LV}+\mathrm{S}$ weight ratio $(\mathrm{RV} / \mathrm{LV}+\mathrm{S})$ (Fulton Index).

\subsection{Immunohistochemical and morphometric analysis of pulmonary arteries}

Lung tissues were fixed in $10 \%$ formalin and paraffin-embedded. The paraffin-embedded tissues from the left lungs were sectioned to yield $3 \mu \mathrm{m}$ thick sections for immunohistochemical analysis and examination by light microscopy. The sections were placed on the Ventana automated stainer BenchMark ULTRA ${ }^{\mathrm{TM}}$ ICH system (Ventana Medical Systems Tucson, AZ). The Ventana staining procedure included dewax antigen retrieval with cell conditioner 1 and incubation with primary antibody (actin smooth muscle clone 1A4, Roche Diagnostic) for $30 \mathrm{~min}$ at $37^{\circ} \mathrm{C}$. For chromogenic detection, ultraView Universal RED detection kit (Ventana, Tucson, AZ) was used. Nuclei were counterstained with Mayer's Hematoxylin. Subsequently, slides were washed in water with a drop of detergent and mounted.

The circumferential actin smooth muscle antibody positive staining around vessels reveals the medial area, which represents the area between the lamina elastica interna and externa. To assess the type of remodeling of muscular pulmonary arteries, vessels were analyzed using a computerized morphometric system (Leica DMD108, Leica Microsystems, Wetzlar, Germany).

In the first analysis, for each animal at least 40 intra-acinar pulmonary vessels of $15-60 \mu \mathrm{m}$ in diameter were selected at a magnification of $\times 100$ in randomly selected fields and evaluated for the degree of muscularization. Each small artery was categorized as: $\mathrm{N}=$ Nonmuscularized (no apparent smooth muscle staining); $\mathrm{P}=$ Partially Muscularized (incomplete medial layer of actin smooth muscle staining) and $\mathrm{M}=$ Muscularized (complete medial layer staining), as previously described (Schermuly et al., 2004). In the second analysis, at least 40 distal pulmonary arteries of $61-200 \mu \mathrm{m}$ in external diameter were divided into two different groups (61-100 $\mu \mathrm{m}$ and $101-200 \mu \mathrm{m})$ and were evaluated for measurement of medial wall thickness at a magnification of $100 \times$. After external diameter and medial thickness measurement of each artery, medial thickness was expressed as follows; percent wall thickness $=[($ medial thickness $\times 2)$ /external diameter $]$ $\times 100$ (Abe et al., 2004). All analyses were done by two observers, blinded to the experimental groups.

\subsection{Pharmacokinetic measurements and analytical methodology}

Lung samples $(0.1 \mathrm{~g}$ tissue $/ \mathrm{ml})$ were homogenised in ammonium formiate $(20 \mathrm{mM}) .50 \mu \mathrm{l}$ of tissue homogenate were added to $200 \mu \mathrm{l}$ of a cold acetonitrile solution containing the Internal Standard (IS) atenolol at concentration of $5 \mu \mathrm{g} / \mathrm{ml}$. After mixing samples were centrifuged for 15 min at $13,000 \times \mathrm{g}\left(5^{\circ} \mathrm{C}\right)$, diluted $1: 1(\mathrm{v} / \mathrm{v})$ with mobile phase $\mathrm{A}$ and injected into LC-MS/MS. Plasma samples were obtained from blood after centrifugation using tripotassium ethylenediaminetetraacetic acid as anticoagulant and processed as follow: $50 \mu \mathrm{l}$ of plasma were added to $200 \mu$ of a cold $\left(4^{\circ} \mathrm{C}\right)$ acetonitrile solution containing the IS at concentration of $5 \mu \mathrm{g} / \mathrm{ml}$. After mixing, samples were centrifuged for $15 \mathrm{~min}$ at $800 \times \mathrm{g}\left(5^{\circ} \mathrm{C}\right)$, diluted $1: 1(\mathrm{v} / \mathrm{v})$ with mobile phase $\mathrm{A}$ and injected into LC-MS/MS. Study samples were analyzed using an ABSciex API 3200 with TurboIonspray interface in positive-ion multiple reaction-monitoring mode. Each sample was injected sequentially onto the LCMS/MS system using a Shimadzu prominence UFLC system and separated using Gemini Nx $-5 \mu$ C18 110 A $50 \times 2.00 \mathrm{~mm}$ as column. $10 \mathrm{mM}$ Ammonium Bicarbonate in water, pH8, was used as mobile phase A and methanol as mobile phase B (flow rate $0.4 \mathrm{ml} / \mathrm{min}$ ). PK parameters and PK simulations were obtained using Phoenix WinNonlin (Pharsight Corporation, Sunnyvale, CA) version 6.2.1. Calibration curves and quality control samples for plasma and lung were prepared using the same procedure of study samples.

\subsection{Statistical analysis}

All values are mean \pm standard error (S.E.M.) of number (n) subjects as indicated in the figures. Statistical analysis was performed using one- or two-way analysis of variance (ANOVA) followed by Dunnett's or Bonferroni post-hoc test for multigroup comparisons by using Prism software (Graph Pad Software, San Diego, CA). P $<0.05$ was considered a level of statistical significance.

\section{Results}

\subsection{Proposed binding mode of Compound 3 within ROCK-1}

To investigate the binding mode of Compound 3, docking simulations were conducted using the X-ray structure of human ROCK-1 (PDB 2ETK) (Jacobs et al., 2006). The best-scored pose of Compound 3 


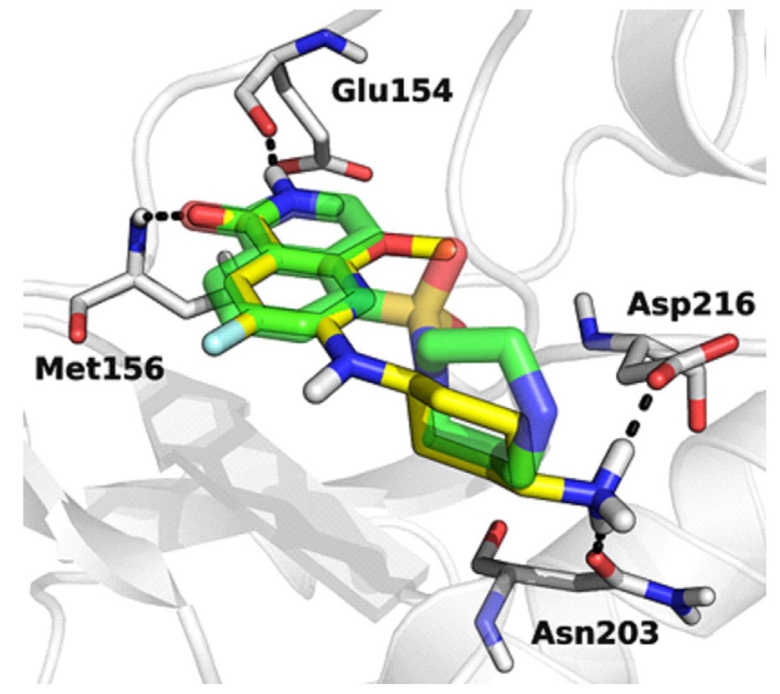

Fig. 2. Docking of Compound 3 in the $x$-Ray structure of hydroxyfasudil bound to human ROCK-1. Compound 3 (yellow) and hydroxyfasudil (green). (For interpretation of the references to color in this figure legend, the reader is referred to the web version of this article.)

superimposed well with the co-crystallized hydroxyfasudil molecule (Fig. 2). Compound 3 bound to the hinge region through its carboxamide group, forming two hydrogen bond interactions with the backbone of Glu154 and Met156 (Fig. 2). Such bidentate interaction was further strengthened by the presence of an intramolecular hydrogen bond between the methoxy oxygen and the neighboring amide $\mathrm{NH}_{2}$ (Guo et al., 2016), yielding to the formation of a pseudo bicyclic system that superimposed well with the isoquinolone ring system of hydroxyfasudil. Besides the hinge interactions, Compound 3 formed extensive hydrophobic contacts with Ile82, Val90, Leu205 and Phe368 through its pyridine ring (not shown in Fig. 2), while the positively charged 4trans-aminocyclohexyl moiety bound to the side chain amide of Asn203 and Asp216.

\subsection{Inhibition of ROCK-1 and ROCK-2 enzymatic activity}

Enzymatic inhibition potency $\left(\mathrm{IC}_{50}\right)$ for Compound 3 were determined for both human isoforms of ROCK-1 and ROCK-2 (Table 1). Compound 3 is a nanomolar inhibitor on both ROCK isoforms, and its measured IC $_{50}$ for ROCK-1 $(2 \mathrm{nM})$ is in line with that reported by Terasawa et al. (2010). ATP competitive behavior of Compound 3 was inferred by examining its binding mode, which resulted very similar to other ATP competitive ROCK inhibitors. In conclusion, Compound 3 resulted about ten and sixty times more potent than Y-27632 and fasudil, respectively.

\subsection{Kinase selectivity}

Compound 3 was counter-screened in a competitive assay against a

Table 1

Inhibitory potencies $\left(\mathrm{IC}_{50}\right)$ of Compound 3, fasudil and Y-27632 against enzymatic activity of human ROCK isoforms 1 and 2 . Where $n=4$, the values are means \pm standard error of four independent experiments performed in triplicate. Where $\mathrm{n}=1$, the values are means and confidence intervals of three determinations in a single experiment.

\begin{tabular}{lll}
\hline & ROCK-1, IC $50(n M)$ & ROCK-2, IC ${ }_{50}(n M)$ \\
\hline Compound $3(n=4)$ & $10 \pm 3.1$ & $7.8 \pm 0.5$ \\
Y-27632 $(\mathrm{n}=1)$ & $109(193-24)$ & $90(116-62)$ \\
fasudil $(\mathrm{n}=1)$ & $844(1174-515)$ & $691(893-489)$ \\
\hline
\end{tabular}

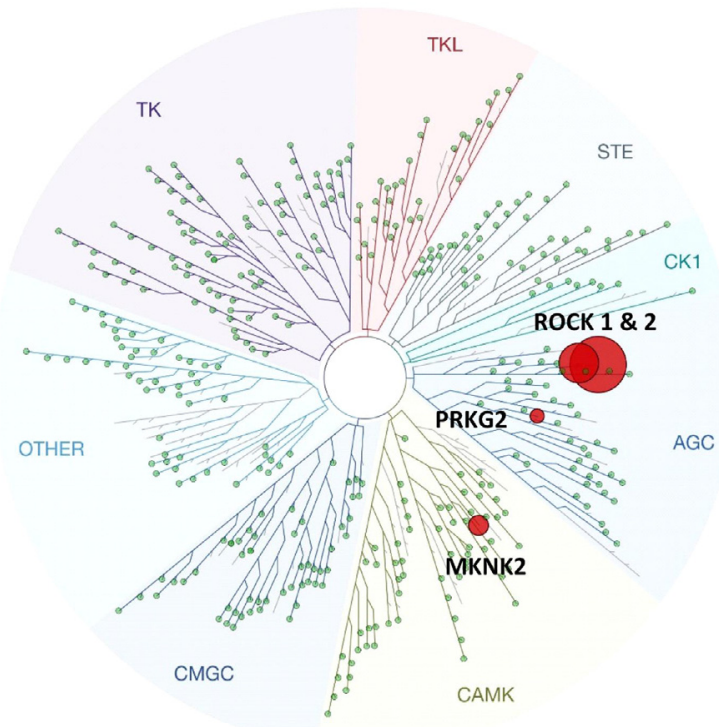

Fig. 3. Dendrogram representation of the human kinome and kinase hits (red dots) by Compound 3 at $100 \mathrm{nM}$, demonstrating kinase selectivity through a KINOMEscan competition binding assay over a large panel (436) of human kinases. Red dots represent interactions with residual activity below $35 \%$ ( $>65 \%$ inhibition). (For interpretation of the references to color in this figure legend, the reader is referred to the web version of this article.)

large panel ( $>400$ ) of human kinases, (Kinome Scan ${ }^{\circledR}$, Discoverx) at the concentration of $100 \mathrm{nM}$, about $10 \times$ times higher than enzymatic $\mathrm{IC}_{50}$ on both isoforms. The graphical view of kinome scan is reported in Fig. 3. Only two hits were detected by setting the threshold for residual activity below $35 \%$, potentially indicating off-target interactions. The hit in the CAMK family is the MKNK2 and the hit in the AGC family is the PRKG2, with $7.8 \%$ and $30 \%$ of residual activity, respectively. For both off-target kinases, the percentage of residual activity is higher than that for ROCK-1 and ROCK-2, $0.05 \%$ and $0.25 \%$, respectively.

\subsection{Vasorelaxant effects in isolated rat pulmonary artery and aortic rings}

The vasorelaxant activity of Compound 3, fasudil and Y-27632 was assessed on rat pulmonary artery pre-contracted with PHE $(1 \mu \mathrm{M})$. The three ROCK inhibitors tested produced relaxation in a concentrationdependent manner, with Compound 3 showing an $\mathrm{IC}_{50}$ value of $51.7 \pm 9 \mathrm{nM}$, resulting more than 10 -fold active respect $\mathrm{Y} 27632$ and fasudil (Fig. 4A). Similarly, in aortic rings pre-contracted with PHE $(1 \mu \mathrm{M})$, Compound 3 fully reversed contraction with an $\mathrm{IC}_{50}$ value of $45.5 \pm 1.1 \mathrm{nM}$, showing a $>30$-fold higher potency in comparison with Y27632 and fasudil, respectively (Fig. 4B). When tested in endothelium-deprived aortic rings, Compound 3 showed a potency IC $_{50}$ $=50.9 \pm 5.2$ ) comparable to that observed when tested in endothelium-intact rings (Fig. 4C).

\subsection{Pharmacokinetic}

The pharmacokinetic profile of Compound 3 after subcutaneous administration of a single dose to rat is shown in Fig. 5. Compound 3 is retained in the lung showing higher concentrations than in plasma probably due to the high volume of distribution of the compound. A compartmental PK model was used to simulate lung concentration-time profile after multiple dose administration using Phoenix WinNonlin software. All PK parameters were fitted to the observed pharmacokinetics on Day 1 and then the lung concentrations were predicted for a twice daily repeated dose administration over 14 days. The 14-day dose simulation in the lung confirmed that levels of Compound 3 in the 
A

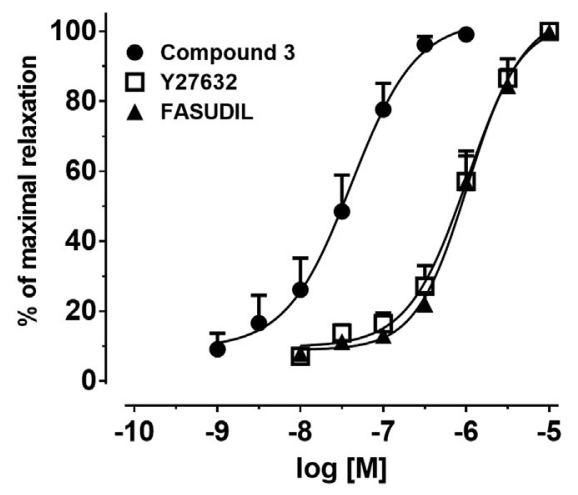

B

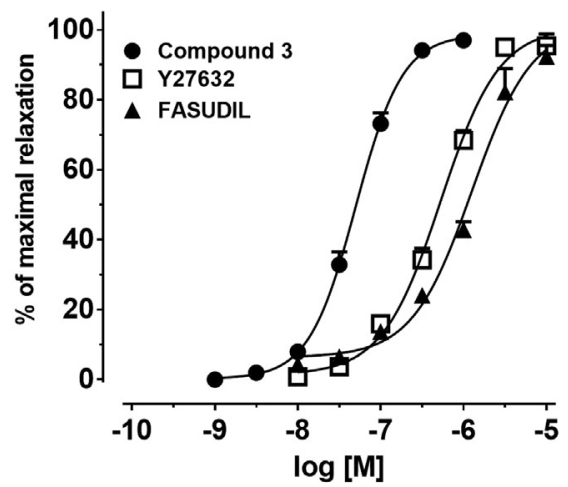

C

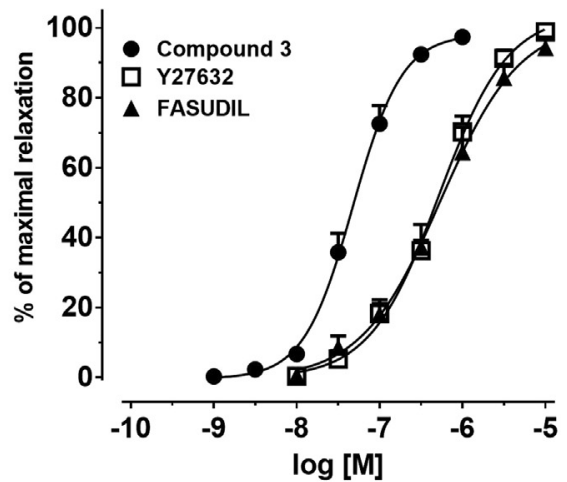

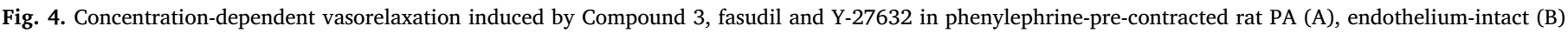

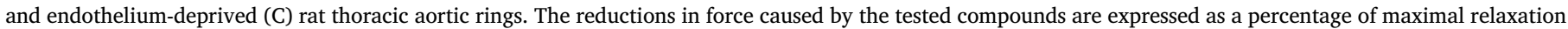
measured at the end of CRC by washing the residual PHE-induced contraction. Each data point represents the mean of 7-8 independent experiments \pm S.E.M.

tissue were constantly at least $>5$-fold above the $\mathrm{IC}_{50}$ value $(45.5 \pm 1.1 \mathrm{nM})$ calculated as vasorelaxant effects in isolated rat aortic rings. Thus, Compound 3 profile proved to be suitable for a twice daily administration without showing significant accumulation upon repeated dosing.

\subsection{Effects in the monocrotaline-induced PAH rat model}

Male Wistar rats were initially randomized into the six groups $(\mathrm{n}=10)$ and treated with a single injection of MCT $(60 \mathrm{mg} / \mathrm{kg} \mathrm{s.c.})$. Fourteen days after MCT injection, two-week interventional treatments with Compound 3 ( 1 or $3 \mathrm{mg} / \mathrm{kg}$, s.c., b.i.d.), macitentan $(10 \mathrm{mg} / \mathrm{kg}$, p.o., q.d.) or respective vehicles (subcutaneous saline, b.i.d. and oral $0.5 \%$ carboxymethyl cellulose in water, q.d.), were initiated. Hemodynamic and gravimetric measurements performed four weeks after MCT induction showed that vehicle-treated rats exhibited severe elevation of RVSP, and right ventricular hypertrophy compared with sham control animals (Fig. 6). All rats belonging to the sham group as well as animals receiving higher dose of Compound $3(3 \mathrm{mg} / \mathrm{kg})$ survived throughout the twenty-eight days after MCT injection. In contrast, in MCT-animals treated with subcutaneous vehicle, lower dose of Compound $3(1 \mathrm{mg} / \mathrm{kg})$, oral vehicle or macitentan $(10 \mathrm{mg} / \mathrm{kg})$ the survival rates were of $70 \%, 80 \%, 80 \%$ and $90 \%$ respectively.

Curative regimens of treatment with Compound 3 or macitentan for two weeks, significantly reduced RVSP compared to MCT group

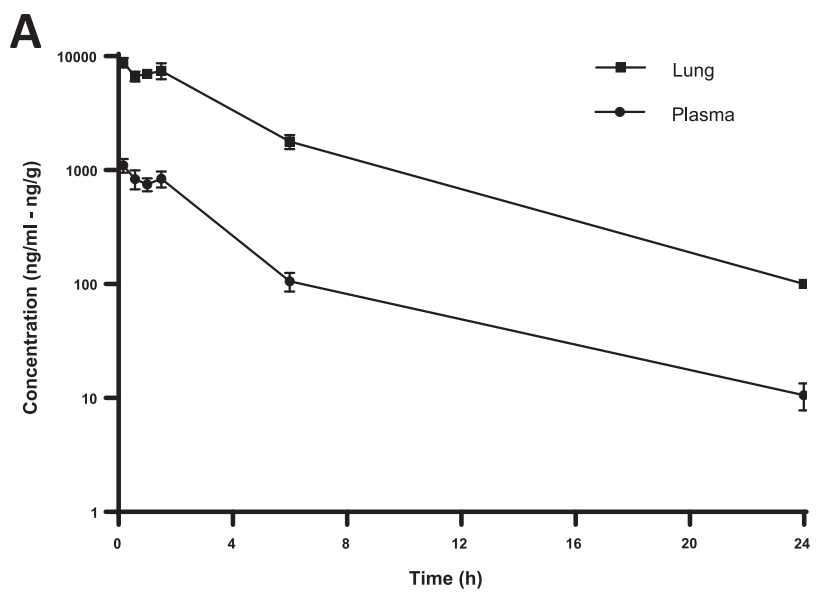

(Fig. 6), without affecting heart rate (Table 2). At day 28 MCT-treated animals showed lower values of SAP compared to healthy animals. While Compound 3 did not affect this value compared to MCT group, treatment with macitentan re-established SAP to normal values.

The RV hypertrophy was evident in MCT rats, showing a significant increased $\mathrm{RV} /(\mathrm{LV}+\mathrm{S})$ mass ratio (Fulton index) compared with the healthy rats $(0.51 \pm 0.03$ vs $0.25 \pm 0.01$; Fig. 6). Treatment with Compound $3 \mathrm{did}$ not reduce the $\mathrm{RV} /(\mathrm{LV}+\mathrm{S})$ ratio in comparison with placebo $(0.49 \pm 0.03$ with $1 \mathrm{mg} / \mathrm{kg}$ dose, $0.49 \pm 0.03$ with $3 \mathrm{mg} / \mathrm{kg}$ dose). Albeit not significant, macitentan showed a trend of reduction in $\mathrm{RV}$ hypertrophy with a mean mass $\mathrm{RV} / \mathrm{LV}+\mathrm{S}$ ratio of $0.41 \pm 0.04$.

The exposure to MCT induced a substantial increase in muscularization in intra-acinar pulmonary vessels $(15-60 \mu \mathrm{m}$ in diameter, Fig. 7). Only $18 \%$ of intra-acinar vessels are fully muscularized in healthy rat lungs and the majority of vessels lacks a muscular layer or presents just a partial muscularization. Conversely, in MCT treated rats, muscularized vessels are the majority while less than $5 \%$ of vessels lack a muscular layer. Treatment with Compound 3 and macitentan showed anti-remodeling effects as evident by the significant reduction in fully muscularized vessels which is accompanied by the increase in nonmuscularized ones (Fig. 7). Next, we quantified medial wall thickness of distal pulmonary arteries subdivided in small and medium size according to the diameter of the lumen: $61-100 \mu \mathrm{m}$ and $101-200 \mu \mathrm{m}$. Compared to healthy group, rat exposed to MCT and treated with vehicles showed a significant increase in medial wall thickness of all the

B

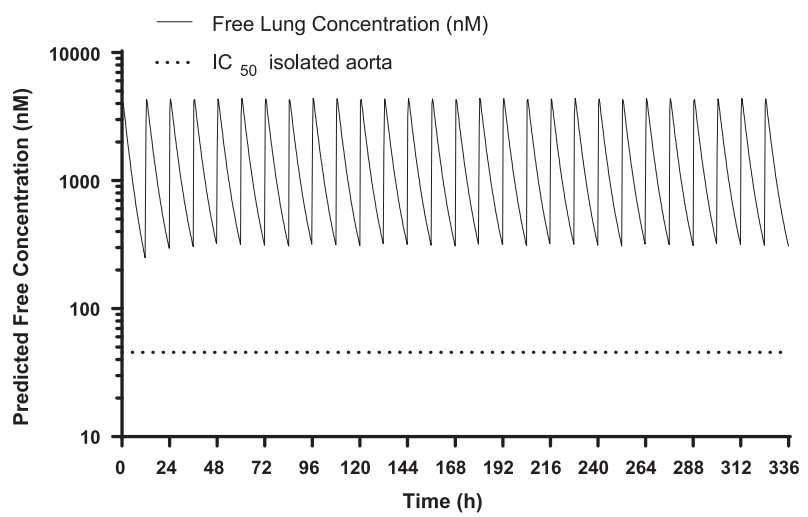

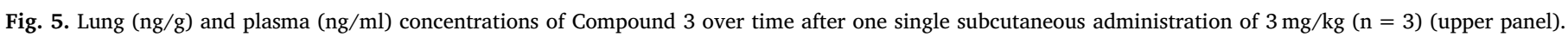

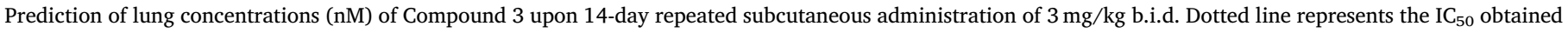
in the isolated pulmonary rat artery vasorelaxation assay (lower panel). 
A

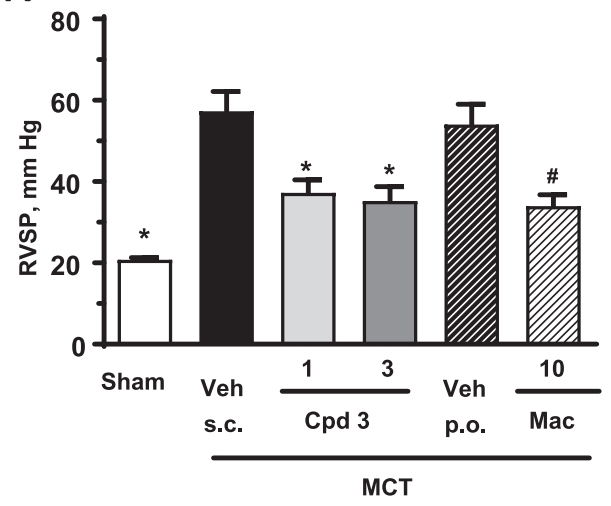

B

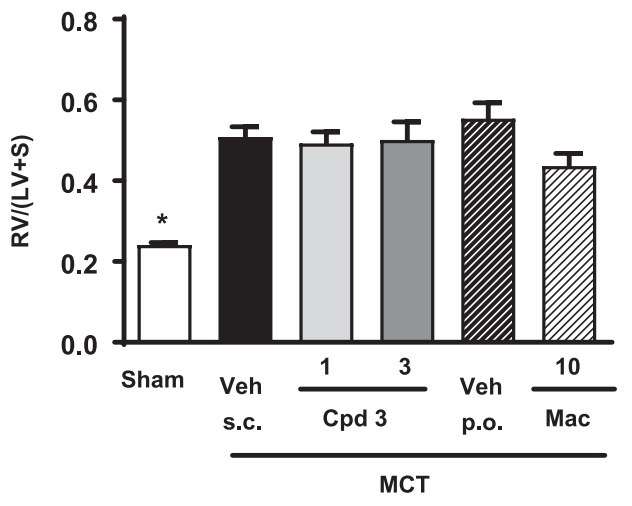

Fig. 6. Effects of 2-week treatment with subcutaneous vehicle (Veh s.c.), Compound 3 (Cpd3, 1 and $3 \mathrm{mg} / \mathrm{kg}$, s.c.), oral vehicle (Veh p.o.) and macitentan (Mac, $10 \mathrm{mg} / \mathrm{kg}$, p.o.) on MCT-induced right ventricle systolic pressure (RVSP) and right ventricle remodeling. RVSP was measured invasively by right heart catheterization (left panel). To determine right ventricular remodeling, the heart was dissected and the ratio of the right ventricle fresh weight to left ventricle plus septum weight $(\mathrm{RV} / \mathrm{LV}+\mathrm{S})$ was calculated (right panel). Each bar represents the mean \pm S.E.M $(n=7-10)$. *p $<0.01$ vs Veh s.c., \#p $<0.01$ vs Veh p.o.

Table 2

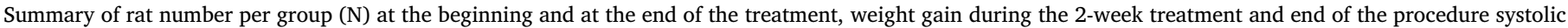
arterial pressure and heart rate measures in the different experimental groups. Values represents the mean \pm S.E.M.

\begin{tabular}{|c|c|c|c|c|c|c|}
\hline & Sham control & MCT + Veh s.c. & MCT + Cpd3, 1 mg/Kg s.c. & $\mathrm{MCT}+\mathrm{Cpd} 3,3 \mathrm{mg} / \mathrm{Kg}$ s.c. & MCT + Veh p.o. & MCT + Mac, $10 \mathrm{mg} / \mathrm{Kg}$ p.o. \\
\hline $\mathrm{N}$ at start & 10 & 10 & 10 & 10 & 10 & 10 \\
\hline $\mathrm{N}$ at end & 10 & 7 & 8 & 10 & 8 & 9 \\
\hline Weight Gain, $g$ & $133 \pm 7^{\mathrm{a}}$ & $74 \pm 7$ & $83 \pm 8$ & $79 \pm 9$ & $89 \pm 8$ & $80 \pm 10$ \\
\hline Systolic arterial pressure, $\mathrm{mmHg}$ & $113 \pm 6^{\mathrm{a}}$ & $81 \pm 6$ & $83 \pm 4$ & $76 \pm 3$ & $97 \pm 5$ & $106 \pm 5$ \\
\hline Heart Rate, bpm & $307 \pm 13$ & $293 \pm 10$ & $301 \pm 13$ & $272 \pm 10$ & $283 \pm 11$ & $303 \pm 8$ \\
\hline
\end{tabular}

a significant vs MCT + Veh s.c. One-way ANOVA, Tukey's multiple comparisons test, $\mathrm{P}<0.01$.

three size groups of arteries (Fig. 7). Both Compound 3 and macitentan significantly reduced the medial thickening of all size groups of arteries, with Compound 3 showing a dose-dependent reduction.

\section{Discussion}

The main findings of this study are: 1 ) Compound 3 is a highly selective ROCK inhibitor and a potent vasorelaxant with an amenable PK profile for subcutaneous delivery; 2) Compound 3 treatment significantly ameliorated hemodynamics and pulmonary vascular remodeling in MCT-induced PAH in the rat; 3) the beneficial effects of Compound 3 are comparable to those obtained, in a head-to-head comparison, with macitentan and were attained with a interventional treatment regimen started when the MCT-induced pathology is considered already progressing.

In the quest for better pharmacological tools for investigating ROCK pathophysiological functions in $\mathrm{PAH}$, we have focused our attention on a novel ROCK inhibitor described as Example 3 in an abandoned patent application (Terasawa et al., 2010) and here indicated as Compound 3. We further proceeded with a thorough characterization of Compound 3 and found that is a potent inhibitor of both ROCK 1 and 2 at nanomolar concentrations.

A remarkable selectivity towards ROCK 1 and 2 was found by spottesting Compound 3 at $100 \mathrm{nM}$ against a large panel of human kinases. A partial explanation for its selectivity toward PKA, which is highly homologue to ROCK, can be provided from docking simulations within ROCK-1 (Jacobs et al., 2006). Indeed, in the best-scored pose of Compound 3 the methoxy group is buried within a small hydrophobic cleft, formed by Met153 and Ala215. This latter residue, is substituted with a threonine in PKA (Thr183). It has already been observed that the increased steric hindrance of this amino acid in PKA compared to ROCK caused a shift in the orientation of Y-27632 within the ATP-binding site, providing a structural clue for the slight selectivity exhibited by this inhibitor towards ROCK-1 (> 30-fold) (Jacobs et al., 2006). Likewise, it can be hypothesized that the threonine side chain in PKA may hamper a proper accommodation of the methoxy group of Compound 3, yielding to a rearrangement of the ligand geometry and the subsequent loss of the key ligand-protein interactions. That said, it cannot be ruled out that the remarkable selectivity exhibited by Compound 3 compared to fasudil and Y-27632 might arise from other complex dynamic mechanisms, including induced-fit effects and/or conformational selection (Agafonov et al., 2014).

By testing Compound 3 in isolated rat pulmonary artery and aortic rings contracted with PHE, we demonstrated that the inhibitory effects observed in the enzymatic assays translate into a robust vasorelaxant activity occurring in the nanomolar range of concentrations. The relaxant effect of Compound 3 is markedly superior to vasorelaxation obtained with fasudil or Y-27632 in head-to head comparisons ( $>30$ fold potency difference).

Together, the in vitro data suggest that Compound 3 is not only a highly selective and potent inhibitor of ROCK but is also a more potent vasorelaxant agent than fasudil and Y-27632. Therefore, we performed chronic treatments in experimental PAH induced by MCT in rats to investigate the therapeutic efficacy of Compound 3. Compound 3 was tested in a head-to-head comparison with macitentan, an orally active, potent, dual endothelin (ET) A and B receptor antagonist currently in use to treat PAH patients. Compared to other endothelin receptor antagonists, macitentan has improved tissue targeting, a longer duration of action and an improved safety profile (DuBrock and Channick, 2014; Iglarz et al., 2015). An interventional treatment regimen was adopted for both macitentan and Compound 3. Treatments started 14 days after MCT induction when the pathology is considered already initiated and rapidly progressing (Nogueira-Ferreira et al., 2015). The mechanism by which MCT treatment leads to PAH is thought to occur initially as an insult to the pulmonary endothelial cells provoking an inflammatory response while smooth muscle medial hypertrophy takes place from about day 14 and increases progressively (Huang et al., 2012; Wilson et al., 2017). For Compound 3, the posology was established based on its pharmacokinetic profile and was twice daily treatment by subcutaneous injection. Macitentan posology was chosen based on existing literature (Iglarz et al., 2008). We found that Compound 3, at both doses tested ( 1 and $3 \mathrm{mg} / \mathrm{kg}$, b.i.d.), ameliorated PAH induced by MCT similarly to macitentan. Interestingly, the lowering of blood pressure was selective for the pulmonary district, as SAP was not significantly 
A
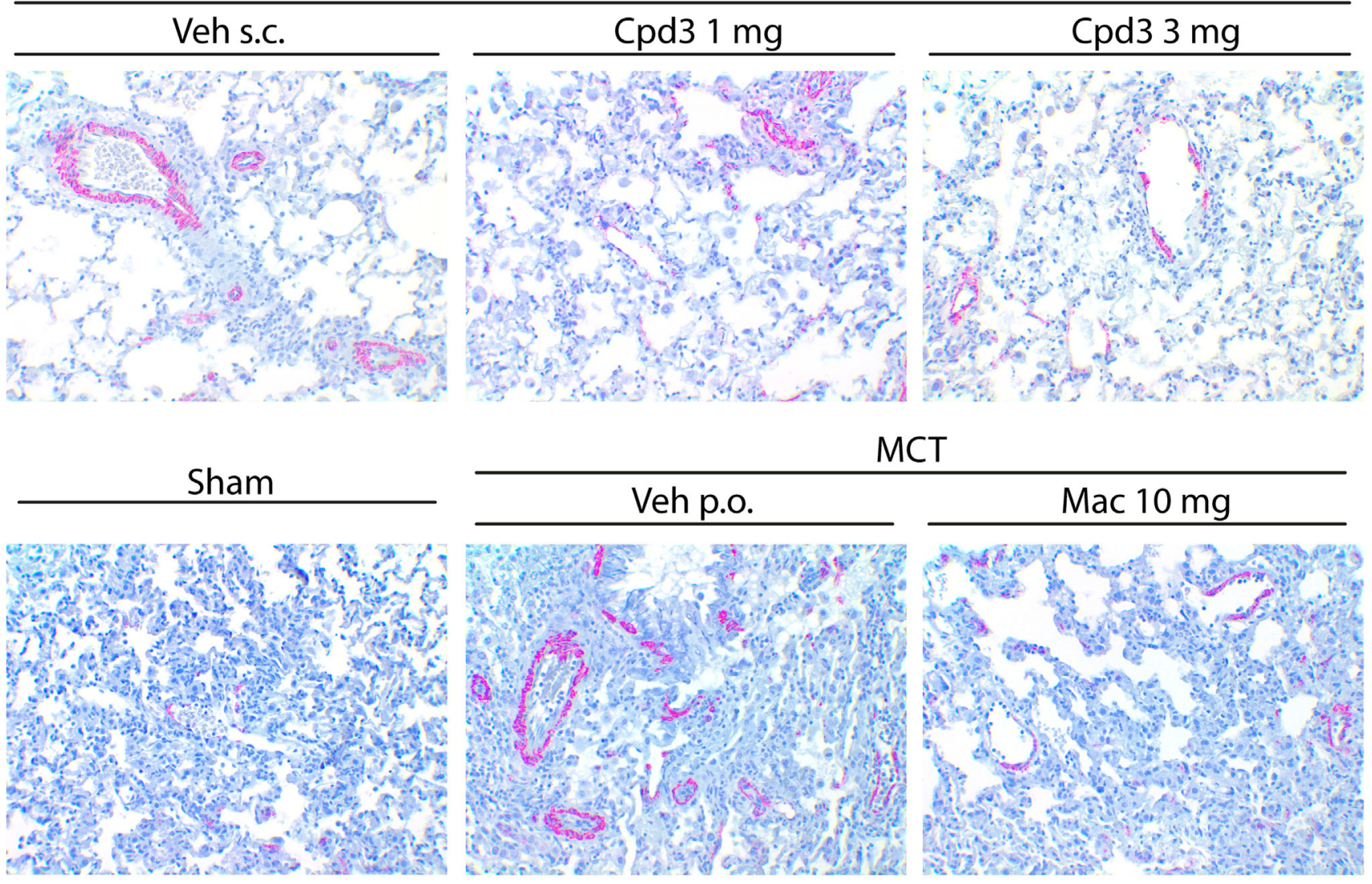

MCT
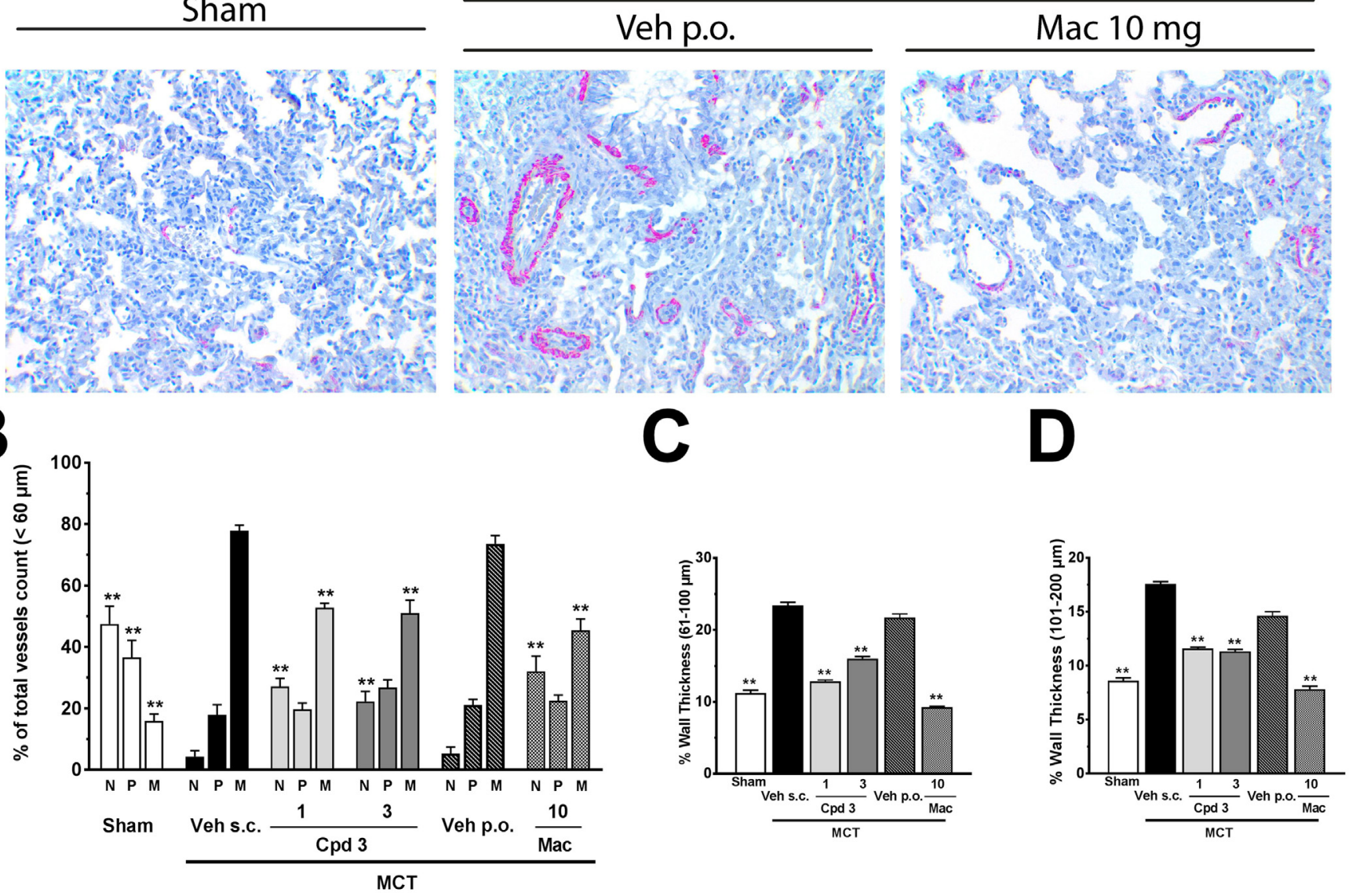

B

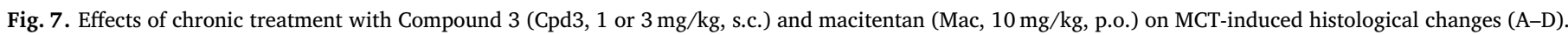

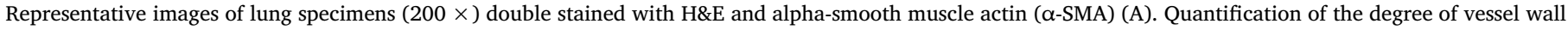

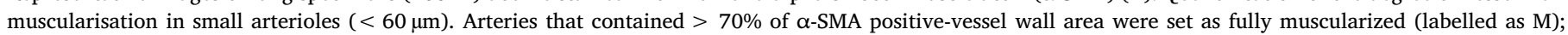

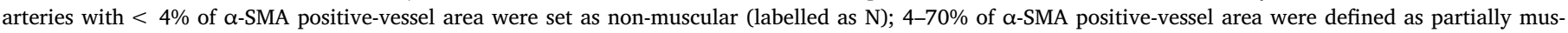

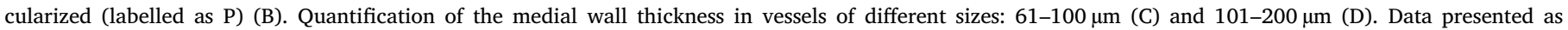
mean \pm S.E.M. ${ }^{*} * \mathrm{P}<0.01$ treatment groups $v$ s. respective vehicles (Veh s.c. or p.o.).

affected in the treated animals. This may appear in contradiction with the blood pressure lowering effect of pharmacological ROCK inhibition reported previously (Kast et al., 2007). However, we must consider that the acute effect on SAP may gradually disappear upon repeated treatments, because of a tolerance phenomenon already known for this class of compounds (Kast et al., 2007). Interestingly, no significant effect was observed on heart rates with any of the treatments adopted, suggesting that Compound 3 treatment did not result in reflex tachycardia. Our finding is in line with a previous study reporting the lack of effect on heart rate of chronic treatment with an oral ROCK inhibitor (azindole1) in experimental PAH (Dahal et al., 2010).

The increase in RV heart weight in the vehicle group is consistent with previous studies in the rat MCT model (Nogueira-Ferreira et al., 2015). We found no protective effect of Compound 3 on RV hypertrophy. In a previous study, fasudil, but not bosentan or sildenafil, was shown to ameliorate RV hypertrophy in the rat MCT model (Mouchaers et al., 2010). The different outcome between the two studies could be explained by the differences in the pathology model. Indeed, 
Mouchaers and colleagues induced PAH with $40 \mathrm{mg} / \mathrm{kg}$ of MCT, a dose inferior to the $60 \mathrm{mg} / \mathrm{kg}$ utilized in this study. It should also be considered that fasudil showed effects unrelated to ROCK inhibition, such as modulation of autophagy (Iorio et al., 2010), which may have an impact in the MCT-induced PAH model. Interestingly, we found that also macitentan did not significantly prevent RV hypertrophy despite a trend. Although a positive effect of macitentan against MCT-induced RV hypertrophy was previously reported, it should be noted that the treatment regimen was preventative as started immediately after MCT injection and lasted for 4 weeks (Iglarz et al., 2015). Thus, a clear effect on RV hypertrophy in the MCT rat model may require preventative treatments or interventional treatments lasting longer than 2 weeks. However, in the MCT model, the disease progression toward death might be too short for compensatory mechanisms to develop. As an alternative, the chronic hypoxia model combined with the VEGFR-2 inhibitor Sugen-5416 (Ciuclan et al., 2011), which is characterized by a slower disease progression could allow longer treatments, thus permitting to further uncover the potential therapeutic effects of ROCK inhibitors in $\mathrm{PAH}$, as well as possible differences in comparison with endothelin antagonists or other established therapies. Although this study was not designed for determining differences in survival between groups, it is interesting to notice that higher mortality was observed in the vehicle-treated group as compared with the group treated with the high dose of Compound 3. This encouraging pro-survival trend may reflect a positive effect of Compound 3 in counteracting disease progression.

Another important finding of this study is that Compound $3 \mathrm{im}$ proved also pulmonary vascular remodeling as evident from significantly reduced muscularization and medial wall thickness of peripheral pulmonary vessels, an effect comparable to that observed in the macitentan-treated group. This anti-remodeling effect may be consequent to improved hemodynamics resulting from vasorelaxation associated with ROCK inhibition. However, induction of apoptosis and reduction of proliferation of pulmonary artery smooth muscle cells (Liu et al., 2011) and inflammatory cell migration (Abe at al, 2004) might also contribute to the attenuation of pulmonary arterial wall remodeling upon pharmacological inhibition of ROCK. A limitation of this study is that important functional cardiac parameters, such as cardiac output, have not been evaluated and further studies in different experimental PAH models will be necessary to prove the therapeutic value of this highly selective ROCK inhibitor. Moreover, we are aware that research on PAH is characterized by significant discordance between preclinical findings and clinical results (Bonnet et al., 2017).

By demonstrating that Compound 3 is capable of counteracting vascular remodeling when treatment is initiated after MCT-induced pathology is already established and rapidly progressing, we provide support to the rationale of developing pharmacological agents targeting ROCK in PAH. Most importantly, we point out to the scientific community that Compound 3 is a versatile, potent and selective pharmacological tool with an amenable pharmacokinetic profile and well tolerated upon chronic treatment.

\section{Acknowledgements}

The authors acknowledge Fabio Rancati, Fabio Dardi, Benedetta Riccardi, Riccardo Patacchini and Maurizio Civelli for scientific inputs and discussions, David Jones and Alex Groarke for performing the enzymatic assays, Christine Edwards, Gurdip Bhalay, Mike Briggs, Mark Withmarsch and scientists at Charles River Laboratories, Harlow, UK, for technical support. This work was funded by Chiesi Farmaceutici, S.p.A., Parma, Italy.

\section{References}

Abe, K., Shimokawa, H., Morikawa, K., Uwatoku, T., Oi, K., Matsumoto, Y., Hattori, T., Nakashima, Y., Kaibuchi, K., Sueishi, K., Takeshit, A., 2004. Long-term treatment with a Rho-kinase inhibitor improves monocrotaline-induced fatal pulmonary hypertension in rats. Circ. Res. 94 (3), 385-393.

Agafonov, R.V., Wilson, C., Otten, R., Buosi, V., Kern, D., 2014. Energetic dissection of Gleevec's selectivity towards human tyrosine kinases. Nat. Struct. Mol. Biol. 21 (10), 848-853.

Asano, T., Ikegaki, I., Satoh, S.-i., Seto, M., Sasaki, Y., 1998. Protein Kinase inhibitor, Fasudil (AT-877): a novel approach to signal transduction therapy. Cardiovasc. Drug Rev. 16 (1), 76-87.

Bonnet, S., Provencher, S., Guignabert, C., Perros, F., Boucherat, O., Schermuly, R.T., Hassoun, P.M., Rabinovitch, M., Nicolls, M.R., Humbert, M., 2017. Translating research into improved patient care in pulmonary arterial hypertension. Am. J. Respir. Crit. Care Med. 195 (5), 583-595.

Budas, G.R., Boehm, M., Kojonazarov, B., Viswanathan, G., Tian, X., Veeroju, S., Novoyatleva, T., Grimminger, F., Hinojosa-Kirschenbaum, F., Ghofrani, H.A., Weissmann, N., Seeger, W., Liles, J.T., Schermuly, R.T., 2018. ASK1 inhibition halts disease progression in preclinical models of pulmonary arterial hypertension. Am. J. Respir. Crit. Care Med. 197 (3), 373-385.

Ciuclan, L., Bonneau, O., Hussey, M., Duggan, N., Holmes, A.M., Good, R., Stringer, R., Jones, P., Morrell, N.W., Jarai, G., Walker, C., Westwick, J., Thomas, M., 2011. A novel murine model of severe pulmonary arterial hypertension. Am. J. Respir. Crit. Care Med. 184, 1171-1182.

Dahal, B.K., Kosanovic, D., Pamarthi, P.K., Sydykov, A., Lai, Y.J., Kast, R., Schirok, H., Stasch, J.P., Ghofrani, H.A., Weissmann, N., Grimminger, F., Seeger, W., Schermuly, R.T., 2010. Therapeutic efficacy of azaindole-1 in experimental pulmonary hypertension. Eur. Respir. J. 36, 808-818.

Dhaliwal, J.S., Badejo, A.M., Casey, D.B., Murthy, S.N., Kadowitz, P.J., 2009. Analysis of pulmonary vasodilator responses to SB-772077-B [4-(7-((3-Amino-1-pyrrolidinyl) carbonyl)-1-ethyl-1H-imidazo(4,5-c)pyridin-2-yl)-1,2,5-oxadiazol-3-amine], a novel Aminofurazan-based Rho Kinase inhibitor. J. Pharmacol. Exp. Ther. 330, 334-341.

Doe, C., Bentley, R., Behm, D.J., Lafferty, R., Stavenger, R., Jung, D., Bamford, M., Panchal, T., Grygielko, E., Wright, L.L., Smith, G.K., Chen, Z., Webb, C., Khandekar, S., Yi, T., Kirkpatrick, R., Dul, E., Jolivette, L., Marino Jr, J.P., Willette, R., Lee, D., Hu, E., 2007. Novel Rho kinase inhibitors with anti-inflammatory and vasodilatory activities. J. Pharmacol. Exp. Ther. 320, 89-98.

DuBrock, H.M., Channick, R.N., 2014. Macitentan for the treatment of pulmonary arterial hypertension. Expert Rev. Respir. Med. 8 (4), 393-399.

Feng, Y., LoGrasso, P.V., Defert, O., Li, R., 2016. Rho Kinase (ROCK) Inhibitors and their therapeutic potential. J. Med. Chem. 59 (6), 2269-2300.

Galiè, N., Humbert, M., Vachiery, J.L., Gibbs, S., Lang, I., Torbicki, A., Simonneau, G., Peacock, A., Vonk Noordegraaf, A., Beghetti, M., Ghofrani, A., Gomez Sanchez, M.A., Hansmann, G., Klepetko, W., Lancellotti, P., Matucci, M., McDonagh, T., Pierard, L.A., Trindade, P.T., Zompatori, M., Hoeper, M., 2015. 2015 ESC/ERS guidelines for the diagnosis and treatment of pulmonary hypertension: the joint task force for the diagnosis and treatment of pulmonary hypertension of the European society of cardiology (ESC) and the European respiratory society (ERS): endorsed by: association for European paediatric and congenital cardiology (AEPC), International Society for heart and lung transplantation (ISHLT). Eur. Respir. J. 46 (4), 903-975.

Gonzales, R.J., Carter, R.W., Kanagy, N.L., 2000. Laboratory demonstration of vascular smooth muscle function using rat aortic ring segments. Adv. Physiol. Educ. 24 (1), 13-21.

Guo, W., Zheng, L.Y., Li, Y.-D., Wu, R.-M., Chen, Q., Yang, D.-Q., Fan, X.L., 2016. Discovery of molluscicidal and cercaricidal activities of 3-substituted quinazolinone derivatives by a scaffold hopping approach using a pseudo-ring based on the intramolecular hydrogen bond formation. Eur. J. Med. Chem. 115, 291-294 (115:291-4).

Hartmann, S., Ridley, A.J., Lutz, S., 2015. The function of Rho-associated Kinases ROCK1 and ROCK2 in the pathogenesis of cardiovascular disease. Front. Pharmacol. 6, 276.

Huang, J., Wolk, J.H., Gewitz, M.H., Mathew, R., 2012. Caveolin-1 expression during the progression of pulmonary hypertension. Exp. Biol. Med. 237 (8), 956-965.

Iglarz, M., Binkert, C., Morrison, K., Fischli, W., Gatfield, J., Treiber, A., Weller, T., Bolli, M.H., Boss, C., Buchmann, S., Capeleto, B., Hess, P., Qiu, C., Clozel, M., 2008 Pharmacology of macitentan, an orally active tissue-targeting dual endothelin receptor antagonist. J. Pharmacol. Exp. Ther. 327 (3), 736-745.

Iglarz, M., Landskroner, K., Bauer, Y., Vercauteren, M., Rey, M., Renault, B., Studer, R., Vezzali, E., Freti, D., Hadana, H., Schläpfer, M., Cattaneo, C., Bortolamiol, C., Weber, E., Whitby, B.R., Delahaye, S., Wanner, D., Steiner, P., Nayler, O., Hess, P., Clozel, M., 2015. Comparison of macitentan and bosentan on right ventricular remodeling in a rat model of non-vasoreactive pulmonary hypertension. J. Cardiovasc. Pharmacol. 66 (5), 457-467.

Iorio, F., Bosotti, R., Scacheri, E., Belcastro, V., Mithbaokar, P., Ferriero, R., Murino, L., Tagliaferri, R., Brunetti-Pierri, N., Isacchi, A., di Bernardo, D., 2010. Discovery of drug mode of action and drug repositioning from transcriptional responses. Proc. Natl. Acad. Sci. USA 107 (33), 14621-14626.

Ishizaki, T., Uehata, M., Tamechika, I., Keel, J., Nonomura, K., Maekawa, M., Narumiya, S., 2000. Pharmacological properties of Y-27632, a specific inhibitor of rho-associated kinases. Mol. Pharmacol. 57 (5), 976-983.

Jacobs, M., Hayakawa, K., Swenson, L., Bellon, S., Fleming, M., Taslimi, P., Doran, J., 2006. The structure of dimeric ROCK I reveals the mechanism for ligand selectivity. J. Biol. Chem. 281, 260-268.

Kast, R., Schirok, H., Figueroa-Pérez, S., Mittendorf, J., Gnoth, M.J., Apeler, H., Lenz, J., Franz, J.K., Knorr, A., Hütter, J., Lobell, M., Zimmermann, K., Münter, K., Augstein, K.H., Ehmke, H., Stasch, J.P., 2007. Cardiovascular effects of a novel potent and highly selective azaindole-based inhibitor of Rho-kinase. Br. J. Pharmacol. 152 (7), 1070-1080.

Li, H., Shin, S.E., Kim, H.W., Kim, H.S., Jung, W.K., Ha, K.S., Han, E.T., Hong, S.H., Choi, I.W., Bae, Y.M., Firth, A.L., Bang, H., Park, W.S., 2016. Y-27632, a Rho-associated 
protein kinase inhibitor, inhibits voltage-dependent $\mathrm{K}+$ channels in rabbit coronary arterial smooth muscle cells. Pharmacology 98 (5-6), 220-227.

Liu, A.J., Ling, F., Wang, D., Wang, Q., Lü, X.D., Liu, Y.L., 2011. Fasudil inhibits plateletderived growth factor-induced human pulmonary artery smooth muscle cell proliferation by up-regulation of p27kip ${ }^{1}$ via the ERK signal pathway. Chin. Med. J. 124 (19), 3098-3104.

Lockette, W., Otsuka, Y., Carretero, O., 1986. The loss of endothelium-dependent vascular relaxation in hypertension. Hypertension 8 (2), II61-II66.

LoGrasso, P.V., Feng, Y., 2009. Rho kinase (ROCK) inhibitors and their application to inflammatory disorders. Curr. Top. Med. Chem. 9, 704-723.

Mouchaers, K.T., Schalij, I., de Boer, M.A., Postmus, P.E., van Hinsbergh, V.W., van Nieuw Amerongen, G.P., Vonk Noordegraaf, A., van der Laarse, W.J., 2010. Fasudil reduces monocrotaline-induced pulmonary arterial hypertension: comparison with bosentan and sildenafil. Eur. Respir. J. 36 (4), 800-807.

Muro T., Seki T., Abe M., Inui J., Sato H., 1990. Preparation of trans-4-aminoalkyl-1(pyridylcarbamoyl)cyclohexanes as calcium blockers. EP 0370498.

Nogueira-Ferreira, R., Vitorino, R., Ferreira, R., Henriques-Coelho, T., 2015. Exploring the monocrotaline animal model for the study of pulmonary arterial hypertension: a network approach. Pulm. Pharmacol. Ther. 35, 8-16.

Oka, M., Fagan, K.A., Jones, P.L., McMurtry, I.F., 2008. Therapeutic potential of RhoA/ Rho kinase inhibitors in pulmonary hypertension. Br. J. Pharmacol. 155 (4), 444-454.

Raja, S.G., 2012. Evaluation of clinical efficacy of fasudil for the treatment of pulmonary arterial hypertension. Recent Pat. Cardiovasc. Drug Discov. 7 (2), 100-104.

Riento, K., Ridley, A.J., 2003. ROCKS: multifunctional kinases in cell behavior. Nat. Rev. Mol. Cell Biol. 4 (6), 446-456.

Satoh, S., Ikegaki, I., Kawasaki, K., Asano, T., Shibuya, M., 2014. Pleiotropic effects of the rho-kinase inhibitor fasudil after subarachnoid hemorrhage: a review of preclinical and clinical studies. Curr. Vasc. Pharmacol. 12 (5), 758-765.

Schermuly, R.T., Ghofrani, M.R.W., Grimminger, F., 2011. Mechanisms of disease: pulmonary arterial hypertension. Nat. Rev. Cardiol. 8, 443-455.

Schermuly, R.T., Kreisselmeier, K.P., Ghofrani, H.A., Yilmaz, H., Butrous, G., Ermert, L., Ermert, M., Weissmann, N., Rose, F., Guenther, A., Walmrath, D., Seeger, W.,

Grimminger, F., 2004. Chronic sildenafil treatment inhibits monocrotaline-induced pulmonary hypertension in rats. Am. J. Respir. Crit. Care Med. 169 (1), 39-45.

Schirok, H., Kast, R., Figueroa-Pérez, S., Bennabi, S., Gnoth, M.J., Feurer, A., Heckroth, H., Thutewohl, M., Paulsen, H., Knorr, A., Hütter, J., Lobell, M., Münter, K., Geiss, V., Ehmke, H., Lang, D., Radtke, M., Mittendorf, J., Stasch, J.P., 2008. Design and synthesis of potent and selective azaindole-based rho kinase (ROCK) inhibitors. ChemMedChem 3, 1893-1904.

Shi, J., Wei, L., 2013. Rho kinases in cardiovascular physiology and pathophysiology: the effect of fasudil. J. Cardiovasc. Pharmacol. 62 (4), 341-354.

Tamura, M., Nakao, H., Yoshizaki, H., Shiratsuchi, M., Shigyo, H., Yamada, H., Ozawa, T., Totsuka, J., Hidaka, H., 2005. Development of specific Rho-kinase inhibitors and their clinical application. Biochim. Biophys. Acta 1754, 245-252.

Terasawa, T., Shigenaga, Itoh, S., Maeda, J., Watanabe, H., Kubo, S., Ishii, N., 2010. Preparation of heterocyclic carboxamide compounds, in particular nicotinamides as rock inhibitors. PCT Int. Appl. WO 2010/0 32875.

Uehata, M., Ishizaki, T., Satoh, H., Ono, T., Kawahara, T., Morishita, T., Tamakawa, H., Yamagami, K., Inui, J., Maekawa, M., Narumiya, S., 1997. Calcium sensitization of smooth muscle mediated by a Rho-associated protein kinase in hypertension. Nature 389, 990-994.

Wilson, D.W., Segall, H.J., Pan, L.C., Dunston, S.K., 2017. Progressive inflammatory and structural changes in the pulmonary vasculature of monocrotaline-treated rats. Microvasc. Res. 38 (1), 57-80. 